\title{
A Study on Burst Error Statistics and Error Modelling for MB-OFDM UWB Systems
}

\author{
Chengxiang Wang and Dayong Xu \\ Heriot-Watt University \\ School of Engineering \& Physical Sciences \\ Electrical, Electronic and Computer Engineering \\ E-mail: cheng-xiang.wang@hw.ac.uk, dx4@hw.ac.uk
}

\begin{abstract}
Error models characterize the statistics of bursty error sequences encountered in digital wireless channels and are therefore, useful for the cross-layer design of wireless networks. In this paper, target error sequences are generated by computer simulations of multiband orthogonal frequency division multiplexing (MB-OFDM) ultra wideband (UWB) systems. A corresponding generative model is then developed based on a properly parameterized and sampled deterministic process followed by a threshold detector and two parallel mappers. Simulation results indicate that the developed deterministic process based generative model (DPBGM) enables us to approximate very closely the characteristics of target error sequences with respect to all the interested burst error statistics.
\end{abstract}

Keywords - MB-OFDM UWB, burst error statistics, error models, deterministic processes, Markov models.

\section{INTRODUCTION}

UWB techniques have attracted much attention due to the promising advantages such as high data rates, low complexity, and low cost for short range wireless communications. The applications of UWB techniques include wireless personal area networks (WPAN), ad hoc and sensor networks, vehicle to vehicle communications, and radar, only to mention a few [1]. MB-OFDM is one of the two physical layer candidate standards submitted to IEEE 802.15 Task Group 3a [2]. It operates in the unlicensed $3.1-10.6 \mathrm{GHz}$ UWB band and OFDM signals are transmitted employing a frequency hopping (multiband) scheme. Multi-carriers are used in MB-OFDM and each sub-carrier only occupies a bandwidth of $4.125 \mathrm{MHz}$. The underlying channel seen by each sub-carrier in MB-OFDM tends to be much less frequency-selective compared to the channel experienced by impulse radio UWB, which occupies all the available UWB spectrum. An obvious advantage of a MB-OFDM system is the potential to avoid or reduce intersymbol interference, while relaxing the burden of an equalizer.

For the design and performance evaluation of coding schemes as well as higher layer protocols in UWB systems, it is essential to study the statistical properties of the underlying error sequences. This leads to the necessity of developing error models, which aim to describe the statistical properties of bursty error sequences encountered in digital wireless communication channels [3]. Error models can be either descriptive [4] or generative [5]. A descriptive model analyzes the statistical behavior of target error sequences obtained directly from a real digital channel or a computer simulation of the overall communication link. This is, however, often a time-consuming task. A generative model is a parameterized mathematical model capable of generating error sequences of similar statistics as produced by target error sequences [5]. Unlike a descriptive model, a generative model can greatly reduce the computational effort for generating error sequences of any desired length and therefore speed up simulations.

In the literature, generative models can roughly be classified into five categories. The first category uses finite [5][7] or infinite [5] state Markov chains. The well-known model belonging to this category is the simplified Fritchman's model (SFM) [6], which is a finite state Markov model with only one error state. Hidden Markov Models (HMMs) [8] are another important category of generative models. Three other categories of generative models have error generation mechanisms different from traditionally used Markov chains. They are based on chaos equations [9], stochastic contextfree grammars [10], and deterministic processes [11]-[14]. Particularly, deterministic process based generative models (DPBGMs) in [12]-[14] were shown to be able to provide excellent fittings to the desired burst error statistics of target error sequences.

To the best of the authors' knowledge, burst error statistics of error processes in UWB systems have not yet been investigated. Although DPBGMs have successfully been applied to narrowband [11]-[14] and wideband [13], [14] systems, their applicability to UWB systems is still an open problem. The aim of this paper is to fill the gaps. Binary target error sequences are provided by MB-OFDM UWB systems [2] with standard UWB channel models developed by the IEEE 802.15.3a group [15]. The resulting burst error statistics are then analyzed and corresponding DPBGMs are developed. Simulation results show that the developed DPBGMs enable us to approximate closely all the interested burst error statistics of the underlying descriptive models. It is expected that the developed generative models will play a significant role in the cross-layer design of UWB networks.

The rest of the paper is organized as follows. The adopted MB-OFDM UWB system is briefly described in Section II. Section III introduces the definitions of terms and interested burst error statistics related to binary error sequences. The design procedure of the developed DPBGM is addressed in Section IV. Section V presents the comparison of burst error 
statistics of the descriptive model, the developed generative model, and a SFM. Finally, the conclusions are drawn in Section VI.

\section{THE MB-OFDM UWB SYSTEM}

In this section, we briefly introduce the employed MBOFDM UWB transmission system [2], which is regarded as our descriptive model and provides us target error sequences. The MB-OFDM UWB system supports several optional data rates in the range from 55 to $480 \mathrm{Mb} / \mathrm{s}$. In this paper, the 200 $\mathrm{Mb} / \mathrm{s}$ mode was selected.

\section{A. Transmitter}

The block diagram of the system is shown in Fig. 1. The transmitter consists of a punctured convolutional encoder, an interleaver, a quadrature phase shift keying (QPSK) modulator, an OFDM transmitter, and a frequency hopper. Various coding rates are derived from a rate $1 / 3$ convolutional code followed by puncturing. Here, the coding rate of $5 / 8$ is used to get the date rate of $200 \mathrm{Mb} / \mathrm{s}$. A multi-stage block-based channel interleaver is used before modulation. The interleaved coded bits are mapped to QPSK symbols using Gray labelling. The QPSK symbols are optionally repeated in time (in two consecutive OFDM symbols) and/or frequency (two tones within the same OFDM symbol) and grouped to form OFDM symbols, reducing the effective code rate by a factor of 2 or 4 and providing an additional spreading gain for low data rate modes. Each OFDM symbol has 100 data sub-carriers, 12 pilot sub-carriers, and 10 guard sub-carriers. Therefore, the number of total sub-carriers to be used is 122 . Via the 128point inverse fast Fourier transform (IFFT) operation, the time domain signals are generated. Then, 32 and 5 zero samples are appended to the IFFT output at the head and the tail, respectively. The radio frequency (RF) of transmitted OFDM signals hops among the three $528 \mathrm{MHz}$ frequency bands with center frequencies at $3.432,3.960$ or $4.488 \mathrm{GHz}$.

\section{B. IEEE 802.15.3a UWB Channel Model}

The IEEE 802.15.3a UWB channel model [15] is largely based on a Saleh-Valenzuela (S-V) approach [16], which models multipath components in clusters. This is partly due to the fact that a very fine resolution can be provided by the ultra wide bandwidth. Each cluster is assumed to experience independent fading as well as each ray within the cluster. Multipath gain coefficients follow a log-normal or Nakagami distribution. Posisson processes are used to model cluster arrival rates and ray arrival rates, while a negative exponential distribution describes the power delay profile. Besides the above smallscale fading effects, the modelling procedure also takes into account the large-scale fading, which is characterized by the log-normal shadowing.

The UWB channel model parameters were determined by fitting primary channel characteristics, such as mean excess delay and rms delay spread, to those of measurements. Four different scenarios were defined based on various measurement environments, namely channel models 1-4 (CM1-CM4) [15].
CM1 describes a line-of-sight (LOS) scenario with a distance range less than $4 \mathrm{~m}$. CM2 describes a non-LOS situation, still in the same range. CM3 describes a larger distance range 4-10 $\mathrm{m}$ for a non-LOS scenario, while CM4 describes an "extreme non-LOS multipath channel". The means and standard deviations of the log-normal shadowing are identical for all the four models.

\section{Receiver}

The receiver side of the MB-OFDM UWB system consists of a frequency dehopper, an OFDM receiver, a QPSK demodulator, a deinterleaver, and a Viterbi decoder. We assume perfect timing and frequency synchronization. Furthermore, for the system parameters and UWB channel model described above, the suffix can safely be assumed longer than the delay spread of the channel impulse response.

In order to recover transmitted data in each OFDM symbol, the receiver first takes the 128 samples of effective data by detaching the suffix from the received signal. It should be noted that the MB-OFDM system uses a null suffix instead of the usual cyclic suffix, to prevent ripples in the spectrum. Thus, in order to remove the inter-carrier interference at the receiver, suffix samples following the data part are added to the front of the data, which is called the cyclic addition. A FFT is applied to the resulting 128 samples to reconstruct data symbols. For the signals in each sub-carrier, the QPSK demodulation is performed with the aid of channel estimation. The repetitions in the time and frequency domains are combined. Finally, demodulated data are deinterleaved and decoded by using a Viterbi algorithm.

\section{BURST ERROR STATISTICS}

For clarity reasons, in this section we first introduce the definitions of the used terms and interested burst error statistics [13], [14] in relevance to error sequences. An error sequence is simply a binary sequence of ones and zeros, where a " 1 " stands for an error bit and a " 0 " denotes a correct bit.

- A gap is defined as a string of consecutive zeros between two ones, having a length equal to the number of zeros.

- An error cluster is a region where the errors occur consecutively and has a length equal to the number of ones.

- An error-free burst is defined as an all-zero sequence with a length of at least $\eta$ bits, where $\eta$ is a positive integer.

- An error burst is a sequence of zeros and ones starting and ending with a " 1 ", and separated from neighboring error bursts by error-free bursts.

Different burst error statistics were presented in the literature. In this paper, we are interested in the following burst error statistics [13], [14]:

- $G\left(m_{g}\right)$ : the gap distribution (GD), which is defined as the cumulative distribution function (CDF) of gap lengths $m_{g}$.

- $P\left(0^{m_{0}} / 1\right)$ : the error-free run distribution (EFRD), which is the probability that an one is followed by at least $m_{0}$ zeros. 
- $P\left(1^{m_{c}} / 0\right)$ : the error cluster distribution (ECD), which is the probability that a zero is followed by $m_{c}$ or more ones.

- $P_{E B}\left(m_{e}\right)$ : the error burst distribution (EBD), which is the CDF of error burst lengths $m_{e}$.

- $P_{E F B}\left(m_{\bar{e}}\right)$ : the error-free burst distribution (EFBD), which is the CDF of error-free burst lengths $m_{\bar{e}}$.

- $P(m, n)$ : the block error probability distribution (BEPD), which is the probability that at least $m$ out of $n$ bits are in error.

- $\rho(\Delta k)$ : the bit error correlation function (BECF), which is the conditional probability that the $\Delta k$ th bit following an error bit is also in error.

Note that the above burst error statistics will be considered as the performance criteria for the evaluation of the developed generative models in Section V. The BEPD and BECF are in general be regarded as the most important statistics. A better generative model should be able to approximate more closely these important statistics.

\section{THE DPBGM}

The invention of DPBGMs [11]-[14] originates from two aspects. First, the well-known close relationship between the statistics of burst errors and the second order statistics of fading envelope processes indicates the possibility of developing generative models based on fading processes. Second, deterministic fading processes demonstrate advantages when employed as channel models since the model parameters can easily be determined and their implementation on a computer is very efficient. The idea of DPBGMs has already been applied to a coherent QPSK system with a Rayleigh fading channel [11], a FH convolutionally coded Gaussian minimum shift keying (GMSK) transmission system in the presence of Rayleigh fading channels [12], and realistic 2.5G mobile communication systems: Enhanced General Packet Radio Service (EGPRS) [13], [14]. In this section, we will follow the line of [13], [14] and develop a DPBGM suitable for MB-OFDM UWB systems.

\section{A. The Parametrization of the Sampled Deterministic Process}

The first design step of a DPBGM lies in the parametrization of the employed deterministic process based on the known quantities obtained from the target error sequence.

For simplicity, we choose the following time-continuous deterministic process [11]-[14]

$$
\tilde{\zeta}(t)=\left|\tilde{\mu}_{1}(t)+j \tilde{\mu}_{2}(t)\right|
$$

where

$$
\tilde{\mu}_{i}(t)=\sum_{n=1}^{N_{i}} c_{i, n} \cos \left(2 \pi f_{i, n} t+\theta_{i, n}\right), \quad i=1,2 .
$$

Here, $N_{i}$ defines the number of sinusoids, $c_{i, n}, f_{i, n}$, and $\theta_{i, n}$ are called the gains, the discrete frequencies, and the phases, respectively. By using the method of exact Doppler spread (MEDS) [11], the phases $\theta_{i, n}$ are considered as the realizations of a random generator uniformly distributed over $(0,2 \pi)$, while $c_{i, n}$ and $f_{i, n}$ are given by

$$
\begin{gathered}
c_{i, n}=\sigma_{0} \sqrt{\frac{2}{N_{i}}} \\
f_{i, n}=f_{\max } \sin \left[\frac{\pi}{2 N_{i}}\left(n-\frac{1}{2}\right)\right]
\end{gathered}
$$

respectively. Here, $\sigma_{0}$ is the square root of the mean power of $\tilde{\mu}_{i}(t)$ and $f_{\max }$ represents the maximum Doppler frequency.

It is required that the occurence rate $R_{E B}=\mathcal{N}_{E B} / N_{t}$ of error bursts at a given period is equal to the level-crossing rate (LCR) of the sampled deterministic process $\tilde{\zeta}\left(k T_{A}\right)$ at a chosen threshold $r_{t h}$. Here, $\mathcal{N}_{E B}$ denotes the total number of error bursts in the target error sequence of length $N_{t}, T_{t}$ is the total transmission time of the reference system, and $T_{A}$ is the sampling interval. Also, The ratio $\mathcal{R}_{B}$ of the mean length of error bursts to the mean length of error-free bursts is fitted to the ratio of the average duration of fades (ADF) at $r_{t h}$ to the average duration of interfades (ADIF) at $r_{t h}$. Moreover, we must ensure that the sampling interval $T_{A}$ is chosen sufficiently small for the successful detection of most of the level crossings and fading intervals at deep levels, i.e., $r_{t h} \leq 1$.

It has been shown in [13], [14] that the second order statistics with respect to the LCR, ADF, and ADIF of the underlying sampled deterministic process $\tilde{\zeta}\left(k T_{A}\right)$ are fully determined by the parameter vector $\boldsymbol{\Psi}=\left(N_{1}, N_{2}, r_{t h}, \sigma_{0}, f_{\max }, T_{A}\right)$. For our purpose at hand, it is not necessary to include all the elements of the parameter vector $\Psi$ in the design. We can first choose reasonable values for $N_{1}, N_{2}$, and $r_{t h}$, e.g., $N_{1}=9$, $N_{2}=10$, and $r_{t h}=0.09$. According to [13], [14], the rest of the parameters can be calculated as follows

$$
\begin{gathered}
\sigma_{0}=\frac{r_{t h}}{\sqrt{2 \ln \left(1+\mathcal{R}_{B}\right)}} \\
f_{\max }=\frac{\mathcal{N}_{E B}\left(1+\mathcal{R}_{B}\right)}{T_{t} \sqrt{2 \pi \ln \left(1+\mathcal{R}_{B}\right)}} \\
T_{A} \approx \frac{4 \sigma_{0}\left[\exp \left(\frac{r_{t h}^{2}}{2 \sigma_{0}^{2}}\right)-1\right]}{\sqrt{5} \pi r_{t h} f_{\text {max }}} \sqrt{-1+\sqrt{1+10 q_{s} / 3}}
\end{gathered}
$$

where $q_{s}$ is a very small quantity which determines the maximum measurement error of the LCR.

With the resulting parameter vector $\boldsymbol{\Psi}$, a sampled deterministic process $\tilde{\zeta}\left(k T_{A}\right)$ is simulated within the necessary time interval $\left[0, \tilde{T}_{t}\right]$, i.e., $0 \leq k T_{A} \leq \tilde{T}_{t}$. Here, $\tilde{T}_{t}=T_{t} \tilde{N}_{t} / N_{t}$ with $\tilde{N}_{t}$ denoting the required length of the generated error sequence. The total numbers of the generated error bursts $\tilde{\mathcal{N}}_{E B}$ and error-free bursts $\tilde{\mathcal{N}}_{E F B}$ can approximately be estimated from $\tilde{\mathcal{N}}_{E B}=\left\lfloor\frac{\tilde{N}_{t}}{N_{t}} \mathcal{N}_{E B}\right\rfloor$ and $\tilde{\mathcal{N}}_{E F B}=\left\lfloor\frac{\tilde{N}_{t}}{N_{t}} \mathcal{N}_{E F B}\right\rfloor$, respectively. Here, $\mathcal{N}_{E F B}$ denotes the total number of errorfree bursts in the target error sequence and $\lfloor x\rfloor$ stands for the nearest integer to $x$ towards minus infinity, i.e., $\lfloor x\rfloor \leq x$. In this manner, an error burst length generator $\widetilde{\mathbf{E B}}_{\text {rec }}$ with $\tilde{\mathcal{N}}_{E B}$ entries and an error-free burst length generator $\mathbf{E F B}_{r e c}$ with $\tilde{\mathcal{N}}_{E F B}$ entries are derived. 


\section{B. The Mappers}

Due to the fact that the resulting EBD and EFBD still deviate largely from the desired curves after the above design step, we have to develop two appropriate mappers, which map the lengths of generated error bursts and error-free bursts to the corresponding lengths. The purpose of the second design step is to adapt the EBD and EFBD of the generated sequence to the desired ones. For reasons of brevity, the developing procedure is ommitted here and the interested readers are referred to [13], [14].

\section{The Generation of Error Sequences}

The third step of the design procedure is to generate error sequences according to the modified $\widetilde{\mathbf{E B}}_{r e c}$ and $\widetilde{\mathbf{E F B}}_{r e c}$ after the mappers. The generation of error-free bursts is straightforward since each entry of $\widehat{\mathbf{E F B}}_{\text {rec }}$ is simply interpreted as the number of consecutive zeros. For generating error bursts it is convenient to first construct parameter vectors $\widehat{\mathbf{E C G}}_{j}$ $\left(j=1,2, \ldots, \tilde{\mathcal{N}}_{E B}\right)$, which reflect the configuration of each error burst in $\mathbf{E B}_{r e c}$ by listing the corresponding consecutive cluster lengths and gap lengths. To this end, we have to find all vectors $\mathbf{E C G}_{i}$ corresponding to error bursts with length $m_{e}$ in the original error burst length recorder $\mathbf{E B}_{r e c}$. Then, for all error bursts with the same length $m_{e}$ in $\mathbf{E B}_{r e c}$, we assign randomly $\widehat{\mathbf{E C G}} \widetilde{ }_{j}$ from all possible vectors $\mathbf{E C G}_{i}$ With such a vector $\widehat{\mathbf{E C G}}{ }_{j}$, an error burst is generated by combining consecutive error clusters (ones) and gaps (zeros). The resulting error sequence is simply the combination of consecutively generated error bursts and error-free bursts.

In short, the design procedure of the developed DPBGM involves three steps: the parametrization of the sampled deterministic process, the development of two mappers, and the generation of error sequences. We call the first two steps as the simulation set-up phase, while the last step the simulation run phase. It should be noted that, although the simulation setup phase of the developed DPBGM requires relatively long time, the simulation run phase is very fast, since it determines directly error burst and error-free burst lengths instead of bit sequences. The general block diagram of the DPBGM is depicted in Fig. 2.

\section{Simulation Results and Discussions}

In this section, the performance of the developed DPBGM is investigated by comparing its burst error statistics against those of the descriptive model described in Section II.

The coded MB-OFDM UWB system in Fig. 1 was simulated to generate target error sequences of length $N_{t}=10^{7}$ at symbol energy-to-noise ratios $\left(E_{s} / N_{0}\right)$ of $0-10 \mathrm{~dB}$ with the separation of $1 \mathrm{~dB}$. Fig. 3 plots the resulting average bit error rate (BER) of the system versus $E_{s} / N_{0}$. By using the DPBGM described in section IV, binary error sequences of length $\tilde{N}_{t}=1.5 \times 10^{7}$ were produced. Then, we analyze the GDs, EFRDs, ECDs, EBDs and EFBDs with $\eta=400$, BEPDs with $n=125$, and BECFs of the target and generated binary error sequences. For the sake of comparison, the relevant results of a six-state SFM were also presented. The transition probability matrix of a $K$-state SFM is calculated by expressing the EFRD $P\left(0^{m_{0}} / 1\right)$ as the sum of $K-1$ exponentials. In our case, the fitting of $P\left(0^{m_{0}} / 1\right)$ is achieved by using five exponentials. In [11]-[14], it has been shown that no better performance can be obtained from SFMs with more than six states.

For brevity reasons, only the simulation results of the coded MB-OFDM UWB system with the CM1 will be presented here. As an example, we choose the $E_{s} / N_{0}$ of $7 \mathrm{~dB}$, which corresponds to a BER of 0.0545 . Figs. $4-6$ demonstrate the resulting ECDs, BEPDs, and BECFs, respectively, of the descriptive model and both generative models. The GDs, EFRDs, EBDs, and EFBDs of three models are not shown here since they match each other closely. It is clear that all the three curves for the DPBGM have excellent agreements with the target ones. However, relatively large deviations were found for the fittings to the desired ECD, BEPD, and BECF by using the SFM. Both generative models require relatively long time in the simulation set-up phase, but the simulation run phase of the DPBGM is faster than that of the SFM. For generating an error sequence of length $1.5 \times 10^{7}$, the DPBGM and SFM need about $77.33 \mathrm{~s}$ and $133.128 \mathrm{~s}$, respectively, for their simulation run phase.

\section{CONCLUSION}

In this paper, we have studied for the first time the burst error statistics of MB-OFDM UWB systems. A corresponding fast binary generative model has been developed by using a properly parameterized and sampled deterministic process followed by a threshold detector and two parallel mappers. The applicability of the developed DPBGM to UWB systems has been well verified by the excellent fitting to the desired burst error statistics. Compared with a widely used SFM, the suggested DPBGM shows a better quality in terms of both the accuracy and efficiency. The developed generative models are expected to be of great importance in the design and performance evaluation of UWB higher layer protocols, especially in the cross-layer optimization of UWB networks.

\section{REFERENCES}

[1] M.Ghavami, L.B.Michael, and R.Kohno, Ultra Wideband Signals and Systems in Communication Engineering. New York: John Wiley \& Sons, 2004.

[2] MBOA-SIG, "MultiBand OFDM physical layer proposal for IEEE 802. 15 Task Group 3a," IEEE P802. 15-O3/268r3, Sept. 2004.

[3] M. Zorzi and R.R. Rao, "Perspectives on the impact of error statistics on protocols for wireless networks," IEEE Personal Commun., vol. 6, no. 10, pp. 32-40, Oct. 1999.

[4] P. M. Crespo, R. M. Pelz, and J. Cosmas, "Channel error profiles for DECT," IEE Proceedings-Commun., vol. 141, no. 6, pp. 413-420, Dec. 1994.

[5] L. N. Kanal and A. R. K. Sastry, "Models for channels with memory and their applications to error control," Proc. of the IEEE, vol. 66, no. 7, pp. 724-744, July 1978

[6] B. D. Fritchman, "A binary channel characterization using partitioned Markov chains," IEEE Trans. Information Theory, vol. 13, no. 2, pp. 221-227, Apr. 1967.

[7] A. Willig, "A new class of packet- and bit-level models for wireless channels," Proc. IEEE PIMRC'02, Lisbon, Portugal, Sept. 2002, pp 2434-2440.

[8] J. G. Frias and P. M. Crespo, "Hidden Markov models for burst error characterization in indoor radio channels," IEEE Trans. Veh. Technol., vol. 46, no. 6, pp. 1006-1020, Nov. 1997 


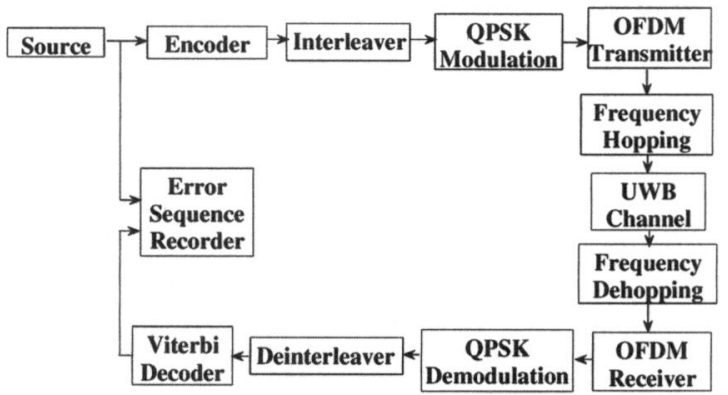

Fig. 1. The block diagram of the MB-OFDM UWB system.

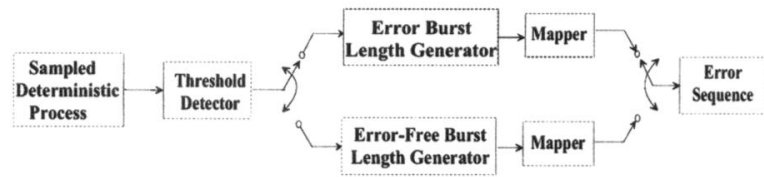

Fig. 2. The block diagram of the developed DPBGM.

[9] E. Costamagna, L. Favalli, and P. Gamba, "Multipath channel modeling with chaotic attractors," Proc. of the IEEE, vol. 90, no. 5, pp. 842-859, May 2002.

[10] W. Zhu and J. G. Frias, "Stochastic context-free grammars and hidden Markov models for modeling of bursty channels," IEEE Trans. Veh. Technol., vol. 53, no. 3, pp. 666-676, May 2004.

[11] C. X. Wang and M. Pätzold, "A generative deterministic model for digital mobile fading channels," IEEE Commun. Letters, vol. 8, no. 4, pp. 223-225, Apr. 2004

[12] C. X. Wang and M. Pätzold, "A new deterministic process based generative model for characterizing bursty error sequences," Proc. IEEE PIMRC'04, Barcelona, Spain, Sept. 2004, pp. 2134-2139.

[13] C. X. Wang, W. Xu, and M. Pätzold, "Error models for evaluating error control strategies in EGPRS systems," Proc. IEEE VTC'04-Fall, Los Angeles, USA, Sept. 2004.

[14] C. X. Wang and W. Xu, "A new class of generative models for burst error characterization in digital wireless channels," IEEE Trans. Commun., submitted for publication.

[15] J. Foerser, "Channel modeling sub-committee report final," IEEE P802. 15-02/368r5-SG3a, Dec. 2002.

[16] A. Saleh and R. Valenzuela, "A statistical model for indoor multipath propagation," IEEE J. Selected Areas in Commun., vol. 5, no. 2, pp. 128137, Feb. 1987.

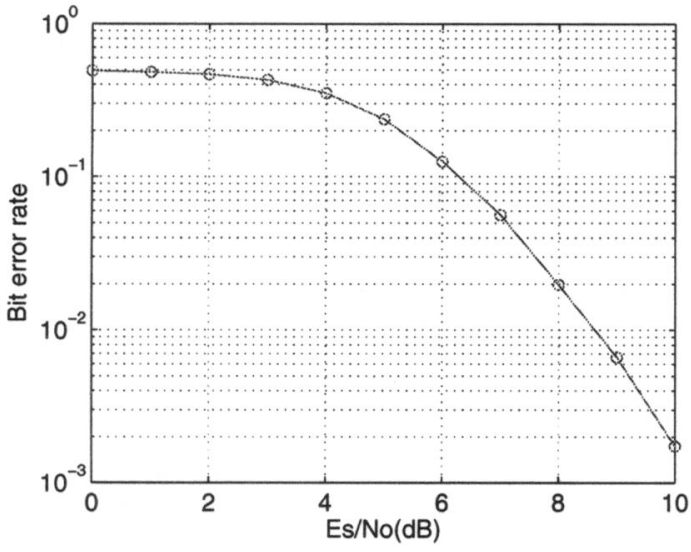

Fig. 3. The BER of the coded MB-OFDM UWB system with CM1.

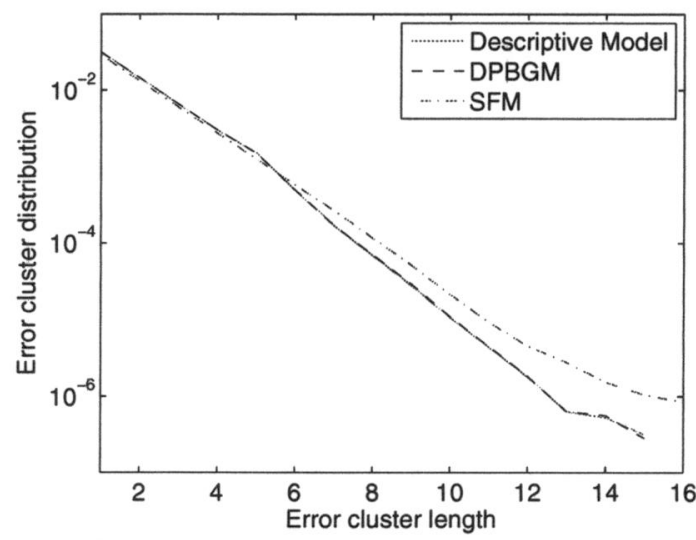

Fig. 4. The ECDs of the descriptive model and the generative models.

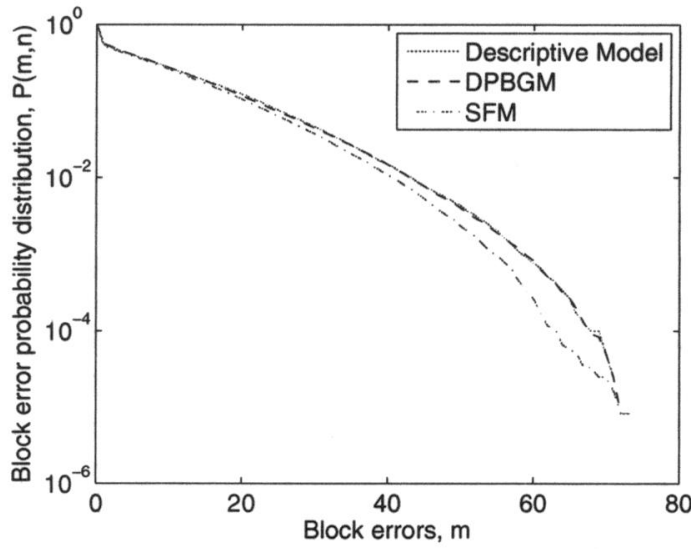

Fig. 5. The BEPDs of the descriptive model and the generative models.

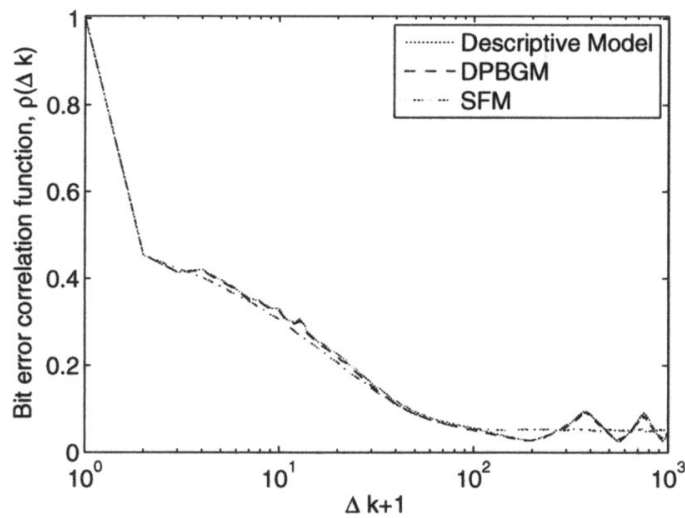

Fig. 6. The BECFs of the descriptive model and the generative models. 